\title{
Deformed Quantum Mechanics and the Landau Problem
}

\author{
J. Gambod and F. Méndet** \\ Departmento de Física, Universidad de Santiago de Chile, Casilla 30\%, Santiago, Chile
}

\begin{abstract}
A deformation of the Landau problem based on a modification of Fock algebra is considered. Systems with the Hamiltonians $f(\hat{H})$ where $\hat{H}$ is the Landau Hamiltonian in the lowest level are discussed. The case $f(\hat{H})=\alpha_{1} \hat{H}+\alpha_{2} \hat{H}^{2}$ is studied and it is shown that in this particular example, parameters of the problem can be fixed by using the quadratic Zeeman effect data and the BreitRabi formula. The proposed approach allows to solve exactly Landau-like families of problems not previously discussed in the literature.
\end{abstract}

In the last years the possibility of deforming quantum mechanics has been widely discussed from different points of view as a way to find a solution to several fundamental problems $1-4]$.

In this regard, issues such as states evolution, algebras of observables and unitarity of the S-matrix have been re-analyzed and, as a consequence, new approaches and concepts in quantum theory have been introduced [5]. It is expected that these new concepts and approaches might shed light on different problems, ranging from the black hole physics ( for example the evaporation process $[6,7])$ to solid state physics (such as an explanation to the high temperature superconductivity [8]).

Although a final answer to these questions has not been reached yet, the methods developed in this field bring different insight and perspectives and they also provide useful calculation techniques to tackle new and old problems. Some of these calculations methods were developed almost at the same time as quantum mechanics. For example, the non-commutativity of spacetime as an ultraviolet regulator [9, 10] or the phase space quantum mechanics 11]. Others, however, such as deformed commutator structures, have led to the development of new and important areas of mathematics such as noncommutative geometry [12], quantum groups [13], and deformed Poissonian geometry [14].

In the present manuscript, we use some of the results outlined before in order to explore a non-commutative system (in the sense of non-commutative quantum mechanics) together with a deformation of commutators (as those appearing in the study of quantum algebras). We focus in a concrete example, namely the Landau problem, and its relation with the quadratic Zeeman effect.

More precisely we will introduce a deformation in the ladder operators - inspired by a modification of Fock algebra - for the Landau problem that emerges from a non-commutative quantum mechanical system and then we will show how this modification might be useful in the understanding the quadratic Zeeman effect and how the free deformation parameters can be set by experiments. In the context of non-commutative quantum mechanics the Landau problem can be understood by defining the operator $\aleph[15]$

$$
\aleph=\frac{\theta^{2}}{4} \mathbf{p}^{2}+x^{2}+y^{2}-\theta\left(x p_{y}-y p_{x}\right),
$$

where $\left\{x, y, p_{x}, p_{y}\right\}$ satisfy the Heisenberg algebra and $\theta$ is a parameter with dimensions of (energy) ${ }^{-2}$. The operator $\aleph$ formally describes a charged two-dimensional harmonic oscillator with mass $m=2 / \theta^{2}$, frequency $\omega=\theta$, in an external magnetic field $B=\theta 1$.

The eigenvalues of $\aleph$ have been discussed in the context of non-commutative quantum mechanics [16] and the important fact is that in a two dimensional noncommutative space, any central field $V\left(|\mathbf{x}|^{2}\right)$ becomes $V(\aleph)$ and therefore, general problems in two dimension can be explicitly addressed.

Let us discuss this last fact. Consider the potential $V\left(|\hat{\mathbf{x}}|^{2}\right)$ in a space where coordinates $\{\hat{x}, \hat{y}\}$ satisfy $[\hat{x}, \hat{y}]=\imath \theta$. By changing the basis of the algebra, this potential satisfies

$$
\begin{aligned}
V\left(\hat{x}^{2}+\hat{y}^{2}\right) & =V\left(\frac{\theta^{2}}{4} \mathbf{p}^{2}+x^{2}+y^{2}-\theta\left(x p_{y}-y p_{x}\right)\right) \\
& =V\left(H_{\mathrm{HO}}-\theta L_{z}\right) \\
& \equiv V(\aleph) .
\end{aligned}
$$

The variables $\left\{x, y, p_{x}, p_{y}\right\}$ satisfy the Heisenberg algebra, the operator $H_{\mathrm{Ho}}$ denotes a two-dimensional harmonic oscillator as in (11), while the operator $L_{z}=\left(x p_{y}-\right.$ $\left.y p_{x}\right)$ is a conserved quantity in the sense $\left[L_{z}, H_{\mathrm{HO}}\right]=0$. This system is an example of non-commutative quantum mechanics and the analysis can be extended to cases where the whole phase space is non-commutative by introducing momentum variables $\left\{p_{x}, p_{y}\right\}$ satisfying $\left[p_{x}, p_{y}\right]=i B$ with $B$ a constant - giving rise to new interesting features such as a sort of phase transition for $\theta=\frac{1}{B}$.

The operator $\aleph$ can be diagonalized in terms of ladder operators. That is, by defining

$$
\begin{aligned}
& a_{ \pm}=\frac{1}{\sqrt{2}}\left(a_{y} \pm i a_{x}\right), \\
& a_{ \pm}^{\dagger}=\frac{1}{\sqrt{2}}\left(a_{y}^{\dagger} \mp i a_{x}^{\dagger}\right) .
\end{aligned}
$$

\footnotetext{
* jorge.gamboa@usach.cl

** fernando.mendez@usach.cl
}

1 Through the paper we use natural units $c=1, \hbar=1$. 
with $a_{x}=(\theta)^{-\frac{1}{2}}\left(x+i(\theta / 2) p_{x}\right)$ and a similar definition for $a_{y}$, being $a^{\dagger}$ the conjugate transposed operator. The operators previously defined satisfy the following algebra

$$
\left[a_{ \pm}, a_{ \pm}^{\dagger}\right]=1, \quad\left[a_{ \pm}, a_{\mp}\right]=0,
$$

and therefore, spaces + and - are orthogonal.

Then, $\aleph$ operator is diagonal in the base $\left|n_{-}, n_{+}\right\rangle$, that is

$$
\aleph\left|n_{-}, n_{+}\right\rangle=\Lambda_{n_{-} n_{+}}\left|n_{-}, n_{+}\right\rangle,
$$

with

$$
\Lambda_{n_{-} n_{+}}=\theta\left(2 n_{-}+1\right),
$$

thus, this is an infinitely degenerate system.

In (7) the $\theta$ parameter emerges as an effective frequency and the factor 1 there is the zero point energy which can be removed by a normal order prescription of operators.

The total Hamiltonian for a particle in this potential reads

$$
H=\frac{1}{2 M} \mathbf{p}^{2}+V(\aleph)
$$

where $M$ is a mass scale. It has been shown 15] that this Hamiltonian is equivalent to the Landau problem in the lowest level - in the strong magnetic field regime - in the limit $M \rightarrow \infty$ for a linear potential $V(\aleph)=\Omega \aleph$ with $\Omega$ a constant with dimensions of (energy) ${ }^{3}$.

Therefore, we study the case in which $M \rightarrow \infty$ and the Hamiltonian turn out to be

$$
H=V(\aleph) .
$$

Since $\aleph$ satisfies (6)

$$
H|n\rangle=V(\aleph)|n\rangle=V\left(\epsilon_{n}\right)|n\rangle,
$$

where $n$ is a notation for the set of quantum numbers $\left\{n_{+}, n_{-}\right\}$and $\epsilon_{n}$ denotes the eigenvalues of $\aleph$.

For $V(\aleph)$ expandable in a Taylor series around a small parameter $\lambda[17$

$$
V(\aleph)=\aleph+\lambda \aleph^{2}+\cdots,
$$

an effective Hamiltonian can be defined

$$
H_{\text {eff }}=: V(\aleph)=\aleph+\lambda \aleph^{2}+\cdots,
$$

and then

$$
H_{\text {eff }}|n\rangle=V\left(\epsilon_{n}\right)|n\rangle .
$$

An example of this system is the Euler-Heisenberg effective Hamiltonian density

$$
\mathcal{H}_{\text {eff }}=\frac{1}{2}\left(\mathbf{E}^{2}+\mathbf{B}^{2}\right)+\xi\left(\mathbf{E}^{2}+\mathbf{B}^{2}\right)^{2}+\cdots,
$$

where $\xi$ in this case in the fine structure constant.

The next step we will take is to make an extra deformation of the above non-commutative system. Indeed, the system previously analyzed admits also a different type of algebra deformation. That is, instead of considering the relation as the one in (5) we posit the relation [18 20]

$$
\left[a_{ \pm}, a_{ \pm}^{\dagger}\right]=\mathcal{D}\left[\lambda_{ \pm} a_{ \pm}^{\dagger} a_{ \pm}\right]
$$

where $\mathcal{D}[x]$ is a deformation operation which, in principle, can be a function of the operator $x$ or an infinite series of $x$ and $\lambda_{ \pm}$is a dimensionless parameter which, in principle, can be chosen as $\lambda_{+}=\lambda_{-}=\lambda$, but we will keep both different in order to consider a more general scenario.

For example if we choose [18] $\mathcal{D}[x]=1-x$, then (15) becomes

$$
a_{ \pm} a_{ \pm}^{\dagger}-q_{ \pm} a_{ \pm}^{\dagger} a_{ \pm}=1
$$

with $q_{ \pm}=1-\lambda_{ \pm}$which turn out to be a representation of the quantum group $S U_{q}(2)$ [13] for each sector '+' and '-'.

The effect of the deformation is to change the spectrum of $a_{ \pm}^{\dagger} a_{ \pm}$and therefore $\Lambda_{n_{-} n_{+}}$changes. Denoting by $c_{n_{-}}\left(\lambda_{-}\right)$the spectrum of the number operator in the '-' sector one gets the spectrum (normal ordered)

$$
\Lambda_{n_{-} n_{+}}=2 \theta c_{n_{-}}\left(\lambda_{-}\right),
$$

where, for the linear deformation $\mathcal{D}[x]=1-x$ [18] one finds [20]

$$
c_{n}(\lambda)=\lambda^{-1}\left(1-(1-\lambda)^{n}\right) .
$$

The infinite degeneracy in $n_{+}$persists since it depends on the fact that $x$ and $y$ in (2) are decoupled sectors. The energy difference between two successive levels for this system turn out to be

$$
\Lambda_{n_{-}+1, n_{+}}-\Lambda_{n_{-}, n_{+}}=2 \theta\left(1-\lambda_{-}\right)^{n_{-}} .
$$

It follows that the spectrum is asymmetric, that is, the spectrum is not equally spaced by $n_{-}$. This is true for any potential of the form $V(\aleph)$.

With this in mind, let us use the fact that $V$ is a function of $p$ and $x$ (or what is the same, $a$ and $a^{\dagger}$ ) in order to study different physical systems. As a concrete example, consider

$$
V(\aleph)=\Omega_{N} \aleph^{N},
$$

with $\Omega_{N}$ a constant with dimensions of (energy) $)^{2 N+1}$. The diagonal basis of operator $a_{ \pm}^{\dagger} a_{ \pm}$is $\left\{\left|n_{ \pm}\right\rangle\right\}_{\left\{n_{ \pm}=0,1,2, \cdots\right\}}$ and due to the properties of the operator $\aleph$, we consider the base $\left|n_{-}, m_{+}\right\rangle$, so that the matrix element (we omit the constant $\Omega_{N}$ )

$\left\langle n_{-}, n_{+}|V| m_{-}, m_{+}\right\rangle=\left\langle n_{-}|V| m_{-}\right\rangle \delta_{n_{+}, m_{+}} \equiv V_{n_{-}, m_{-}} \delta_{n_{+}, m_{+}}$ where the matrix element of the normal ordered operator $V$ is

$$
\begin{aligned}
V_{n_{-}, m_{-}} & =\left\langle n_{-}|\aleph . \aleph \cdots \aleph| m_{-}\right\rangle \\
& =\sum_{\left\{n_{i}\right\}}\left\langle n_{-}|\aleph| n_{1}\right\rangle\left\langle n_{1}|\aleph| n_{2}\right\rangle \cdots\left\langle n_{N}|\aleph| m_{-}\right\rangle \\
& =(2 \theta)^{N}\left(c_{n_{-}}\left(\lambda_{-}\right)\right)^{N} \delta_{n_{-}, m_{-}} .
\end{aligned}
$$


For the particular case of the linear deformation $\mathcal{D}[x]=$ $1-\lambda x$ with $\lambda \equiv \lambda_{-}$we obtain

$$
V_{n_{-}, m_{-}}=\left(\frac{2 \theta}{\lambda}\right)^{N}\left[1-(1-\lambda)^{n_{-}}\right]^{N} \delta_{n_{-}, m_{-}} .
$$

For the case of Hamiltonian (8), this result implies

$$
\begin{gathered}
\left\langle n_{-}, n_{+}|H| m_{-}, m_{+}\right\rangle=\left\langle n_{-}, n_{+}\left|\frac{\mathbf{p}^{2}}{2 M}\right| m_{-}, m_{+}\right\rangle+ \\
\Omega_{N}\left(\frac{2 \theta}{\lambda}\right)^{N}\left[1-(1-\lambda)^{n_{-}}\right]^{N} \delta_{n_{-}, m_{-}} \delta_{n_{+}, m_{+}}
\end{gathered}
$$

However, since we are considering the lowest Landau level limit, the spectrum (note that the change of notation $\left.n_{-} \equiv n\right) E_{n} \sim V_{n, n}$ becomes

$$
E_{n}=\Omega_{N}\left(\frac{2 \theta}{\lambda}\right)^{N}\left[1-(1-\lambda)^{n}\right]^{N} .
$$

Note that this energy is basically the energy of the turning points (which further highlights the non perturbative character of this result).

In order to make contact with the Landau problem described before we take $\Omega_{N}=\Omega^{N}$ so that the identification $\Omega \theta=e H_{0} / 2 \mu$ holds. Here, $e$ is the electron charge, $\mu$ is a mass scale and $H_{0}$ is an external magnetic field.

For $N=1$ (in the limit $M \rightarrow \infty$ ) this model defines a deformed Landau problem. The energy can be written, alternatively

$$
\begin{aligned}
E_{n} & =2 \Omega \theta \sum_{\ell=0}^{n-1} \frac{n(n-1) \cdots(n-\ell)}{(\ell+1) !}(-\lambda)^{\ell} \\
& =2 \Omega \theta n\left(1-\frac{n-1}{2 !} \lambda\right)+\mathcal{O}\left(\lambda^{2}\right),
\end{aligned}
$$

showing that corrections to the energy due to the deformation is order $n^{2}$. Similar behavior is observed in the non-relativistic limit of the relativistic Landau problem, however, relativistic corrections there are also proportional to $(\Omega \theta)^{2}$.

This suggest that a higher orders of the $\aleph$ operator might be of physical interest. Consider, for example, the potential

$$
V^{(2)}=\Omega \aleph+\frac{\kappa^{2}}{4} \Omega^{2} \aleph^{2},
$$

where $\kappa^{2}$ is a length scale which is determined below by using the quadratic Zeeman effect data and the BreitRabi formula.

According to our previous analysis and also under same assumptions, the energy spectrum turn out to be

$$
\begin{aligned}
E_{n}^{(2)}= & \left(\frac{e H_{0}}{2 \mu}\right) n((2+(1-n) \lambda)+ \\
& \left(\frac{e H_{0}}{2 \mu}\right)^{2} \kappa^{2} n^{2}(1+(1-n) \lambda)+\mathcal{O}\left(\lambda^{2}\right) .
\end{aligned}
$$

We observe here that linear terms in $\lambda$ has contributions from linear and quadratic terms of magnetic field with different powers of $n$ and

$$
\begin{aligned}
\Delta E_{n}^{(2)}= & E_{n}^{(2)}-E_{n-1}^{(2)} \\
= & 2\left(\frac{e H_{0}}{2 \mu}\right)(1+(1-n) \lambda)+ \\
& \kappa^{2}\left(\frac{e H_{0}}{2 \mu}\right)^{2}\left[2 n-1+\left(5 n-2-3 n^{2}\right) \lambda\right]
\end{aligned}
$$

For non-highly excited levels, the difference of energies becomes

$\Delta E_{n}^{(2)} \approx 2\left(\frac{e H_{0}}{2 \mu}\right)(1+(1-n) \lambda)+\kappa^{2}\left(\frac{e H_{0}}{2 \mu}\right)^{2}\left(2 n-1-3 n^{2} \lambda\right)$.

In the conventional Landau problem, the difference of frequencies is a constant proportional to the external applied magnetic field. In the case we are considering, the deformation takes an extra factor proportional to $\kappa^{2}\left(\frac{e H_{0}}{2 \mu}\right)^{2}$ and then, if the magnetic field is large enough, and using the condition that $\lambda$ can be properly adjusted, this correction of frequency could be relevant. To be precise, it is enough to have

$$
H_{0} \gg\left(\frac{2 \mu}{e \kappa^{2}}\right) \frac{(1+(1-n) \lambda)}{\left(2 n-1-3 n^{2} \lambda\right)} \sim\left(\frac{2 \mu}{e \kappa^{2}}\right) \frac{1}{3 n^{2}} .
$$

Actually the $H_{0}^{2}$ contribution due to the deformation plays a similar role of the quadratic Zeeman effect term. In our case, for strong magnetic field as described before, one have

$$
\begin{aligned}
\left|\Delta E_{n}^{(2)}\right| & \approx H_{0}^{2}\left(\frac{\kappa e}{2 \mu}\right)^{2} 3 n^{2} \lambda \\
& \equiv \kappa_{\text {eff }} H_{0}^{2}
\end{aligned}
$$

Although the quadratic corrections of the Zeeman effect are not dominant in atomic physics in general, they are important, for example in the case of alkaline atoms where

$$
|\Delta \omega|=\kappa_{\mathrm{eff}} H_{0}^{2} .
$$

These quadratic corrections have been measured in the last twenty years using different methods and great precision has been achieved with the development of cold atoms measurement techniques [22]. For example for the ${ }^{87} \mathrm{Rb}$ ground-state clock transition $\kappa$ is

$$
\kappa_{\text {eff }} \sim 575,15 \times 10^{8} \mathrm{~Hz} \mathrm{~T}^{-2},
$$

but from the the Breit-Rabi formula [21]

$$
\Delta \omega=\frac{\left(g_{J}-g_{I}\right) \mu_{B}^{2}}{2 h \Delta H_{H f s}} H_{0}^{2}=\kappa_{\mathrm{eff}} H_{0}^{2},
$$

where $g_{J, I}$ are the Landé factors, $\mu_{B}$ the Bohr magneton, $h$ the Planck constant and $\Delta H_{H f s}$ is the hyperfine energy splitting. 
In particular the experimental value (34) compared to the $\kappa$ calculated with the Breit-Rabi formula is in excellent agreement with standard measurements [23, 24].

Putting $\kappa$ in natural units then the dimensions of $\kappa$ are

$$
\kappa \sim(\text { length })^{3}
$$

and replacing (34) we find

$$
\kappa^{1 / 3} \sim 0.1 \stackrel{\circ}{A}
$$

which typically could be considered an x-ray regime effect.

\section{CONCLUSIONS}

In this work we have studied a linear deformation of the Fock algebra and we have applied it to the Landau pro- blem which leads to a family of exactly soluble problems. We consider in detail the quadratic effective Hamiltonian and the length scale is fixed using data from the quadratic Zeeman effect.

A careful analysis of (29) shows that the validity of our arguments between neighboring levels as predicted by the Breit-Rabi formula 2 .

The uncertainties of the Breit-Rabi formula are uncertainties in the physical and atomic constants so that the only possible sources of error may come from the g-factors, but the data considered (cold alkaline atoms [23]) seem to be well enough established.

The case of linear potential, on the other hand, can be solved completely and its extension to the relativistic case is straightforward and is equivalent to the formulation of non-commutative fields as it was discussed in [25].

We would like to thank Prof. José Luis Cortes for enlightening discussions. This work was partially supported by Dicyt 041831GR (J.G) and 041931MF (F. M.).
[1] T. Banks, L. Susskind and M. E. Peskin, Nucl. Phys. B 244, 125 (1984).

[2] R. Penrose, in General Relativity; an Einstein Centenary Survey, pp. 581, edited by W. Israel and S. W. Hawking, Univ. Cambridge Press (1979).

[3] T. W. B. Kibble, Commun. Math. Phys. 65, 189 (1979).

[4] S. Weinberg,Annals Phys. 194, 336 (1989).

[5] For recents references see, T. Banks, arXiv:2001.07662 [hep-th]]; L. Amadei, H. Liu and A. Perez, arXiv:1912.09750 [gr-qc]]; S. B. Giddings, Phil. Trans. Roy. Soc. Lond. A 377 (2019) no.2161, 20190029; A. O. Barvinsky, D. Carney and P. C. E. Stamp, Phys. Rev. D 98 (2018) no.8, 084052.

[6] G. T. Horowitz and J. Polchinski, Phys. Rev. D 55, 6189 (1997).

[7] See for example, T. Banks, L. Susskind and M. E. Peskin, Nucl. Phys. B 244, 125 (1984).

[8] A. Das, J. Gamboa, F. Méndez and F. Torres, Phys. Lett. A 375, 1756 (2011).

[9] H. S. Snyder, Phys. Rev. 71, 38 (1947).

[10] C. N. Yang, Phys. Rev. 72, 874 (1947).

[11] T. Curtright, T. Uematsu and C. K. Zachos, J. Math. Phys. 42 (2001), 2396; T. L. Curtright and C. K. Zachos, Asia Pac. Phys. Newslett. 1 (2012), 37-46; T. Curtright, D. Fairlie and C. K. Zachos, Phys. Rev. D 58 (1998), 025002

[12] A. Connes, Noncommutative Geometry, Academic Press, 1994.

[13] Drinfel'd, V.G. J Math Sci 41, 898 (1988); M. Chaichian and P. Kulish, Phys. Lett. B 234, 72 (1990); A. J. Macfarlane, J. Phys. A 22, 4581 (1989); L. C. Biedenharn, J. Phys. A 22, L873 (1989).

[14] M. Kontsevich, Lett. Math. Phys. 66, 157 (2003); ibid.
M. Kontsevich, Commun. Math. Phys. 147, 1 (1992); F. Bayen, M. Flato, C. Fronsdal, A. Lichnerowicz and D. Sternheimer, Annals Phys. 111, 61 (1978); A. S. Cattaneo and G. Felder, Commun. Math. Phys. 212, 591 (2000).

[15] J. Gamboa, M. Loewe, F. Mendez and J. C. Rojas, Int. J. Mod. Phys. A 17, 2555 (2002); ibid, J. Gamboa, M. Loewe, F. Mendez and J. C. Rojas, Mod. Phys. Lett. A 16, 2075 (2001).

[16] This is a topic extensively studied in the last twenty years and here we follow our work and other ones see; L. Mezincescu, arXiv:hep-th/0007046 [hep-th]]; J. Gamboa, M. Loewe and J.C.Rojas, Phys. Rev. D 64, 067901 (2001); V. Nair and A. Polychronakos, Phys. Lett. B 505, 267274 (2001); C. Duval and P. A. Horvathy, Phys. Lett. B 479, 284 (2000); C. Duval and P. A. Horvathy, J. Phys. A: Math. Gen. 34, 10097 (2001).

[17] See for example, D. J. Gross, J. Kruthoff, A. Rolph and E. Shaghoulian, Phys. Rev. D 101, no. 2, 026011 (2020) and references therein.

[18] A. Kempf, J. Math. Phys. 35, 4483 (1994.

[19] A. Kempf, J. Phys. A 30, 2093 (1997).

[20] J. L. Cortés and J. Gamboa, "Deformed classicalquantum mechanics transition," , arXiv:2004.05673 [hepth].

[21] G. Breit and I. I. Rabi Phys. Rev. 38, 2082 (1931).

[22] For references and a review see for example, B- M. Garraway and H. Perrin, J. Phys. 49 B, 172902 (2016).

[23] D. A. Steck, 2010 Rubidium 87 D Line Data, http:7steck.us/alkalidata

[24] B. Wu et. al. J. Opt. Soc. Am. B 31, 742 (2014).

[25] J. M. Carmona, J. L. Cortes, A. K. Das, J. Gamboa and F. Mendez, Mod. Phys. Lett. A 21 (2006), 883-892

\footnotetext{
${ }^{2}$ For a recent experimental Breit-Rabi formula verification see [24].
} 